\title{
Cancer risk in the rubber industry: a review of the recent epidemiological evidence
}

\author{
Manolis Kogevinas, Maria Sala, Paolo Boffetta, Neely Kazerouni, Hans Kromhout, \\ Shelia Hoar-Zahm
}

Respiratory and

Environmental Health

Research Unit, Institut Municipal

d'Investigació Mèdica, Barcelona, Spain

M Kogevinas

M Sala

Unit of Environmental Cancer Epidemiology, International Agency for Research on

Cancer, Lyon, France

P Boffetta

Occupational

Epidemiology Branch,

National Cancer

Institut, Bethesda,

USA

N Kazerouni

S Hoar-Zahm

Department of

Environmental and

Occupational Health,

Wageningen

Agricultural

University,

Wageningen, The

Netherlands

$\mathrm{H}$ Kromhout

Correspondence to:

Dr M Kogevinas, Respiratory

and Environmental Health

Research Unit, Institut

Municipal d'Investigació

Mèdica (IMIM), 80 Doctor

Aiguader Road, Barcelona

08003, Spain. Tel: 00343

2211009; Fax: 00343

2213237; Email:

KOGEVINAS@IMIM.ES

Accepted 10 July 1997

\begin{abstract}
Objectives-To examine the recent epidemiological evidence on cancer risk among workers in the rubber industry.

Methods-Epidemiological studies published after the last detailed review by the International Agency for Research on Cancer (IARC) in 1982 were reviewed. 12 cohort studies in nine countries that examined distinct populations of workers in the rubber industry, seven industry based nested case-control studies, 48 community based case-control studies in 16 countries, and 23 studies based on administrative data that reported risks for employment in the rubber industry were identified.

Results-Excess risks of bladder cancer, lung cancer, and leukaemia were found in most studies, with risks above 1.5 in about half of the studies. A moderate excess risk for laryngeal cancer was consistent across studies. Excess risks were found in a few studies for cancers of the oesophagus, stomach, colon, liver, pancreas, skin, prostate, kidney, brain, and thyroid, and for malignant lymphoma and multiple myeloma, but overall results were not consistent for these neoplasms.

Conclusions-Magnitude of the observed risks varied considerably between studies, but overall the findings indicate the presence of a widespread moderate increased cancer risk among rubber workers. The most consistent results were for bladder, laryngeal, and lung cancer and for leukaemia. Excess risks were also found for other neoplasms but an evaluation of the consistency of the findings is difficult because of the possible selective reporting of results. Recent studies do not provide information associating specific exposures with cancer risk. The preventive measures taken in the rubber industry in recent years may decrease risks, but this has not been documented yet in epidemiological studies.
\end{abstract}

(Occup Environ Med 1998;55:1-12)

Keywords: neoplasms; rubber; occupation
The evidence of a carcinogenic risk in the rubber industry has been reviewed by the International Agency for Research on Cancer (IARC) in $1982^{1}$ and in summary form in 1987. ${ }^{2}$ The IARC Working Group ${ }^{1}$ concluded that there was sufficient evidence that employment in this industry entails a carcinogenic risk. This association was considered causal for bladder cancer and leukaemia, whereas confounding could not be ruled out for the excess risks found for stomach and lung cancer. The strength of the evidence was considered to be limited for cancer of the skin, prostate, and colon, and for lymphoma and inadequate for cancer of the brain, thyroid, and pancreas.

Examination of processes or exposures within this industry was pre-empted at the time of the IARC evaluation, because of the limited information available. The early studies of rubber workers in the United Kingdom ${ }^{34}$ showed a large increase in the risk of bladder cancer among workers employed during the 1930s and 1940 s, which was attributed to exposure to $\beta$-naphthylamine. Subsequent studies have shown that removal of this agent led to a decreased cancer risk..$^{5-7}$ It was postulated that heavy exposure to dusts such as carbon black in the first steps of production lines may be associated with the risk of stomach cancer, and that the risk of lung cancer was associated with exposure to curing fumes. In the United States, a large study of rubber workers employed in two companies in Ohio, showed little or no excess of bladder cancer, ${ }^{89}$ whereas there was a suggestion of an increased risk of stomach cancer, possibly related to exposure to talc, ${ }^{10}$ of prostate cancer, ${ }^{11}$ and lymphatic leukaemia, ${ }^{12}$ possibly associated with exposure to solvents, particularly benzene.

Recent exposures in the rubber industry Since the early 1950 s and more extensively since the 1970s, health and safety measures have been widely applied in the rubber industry by substituting some chemical agents and controlling exposure to others. ${ }^{13} \mathrm{~A}$ recent extensive exposure survey in the rubber industry in The Netherlands, did not find the large differences in the past in exposure to airborne particulates between front processing (weighing and mixing, calandaring, extruding) and back processing (curing and vulcanising). 
Table 1 Summary data on cohort studies and studies based on asministrative data in the rubber industry published after 1982

\begin{tabular}{|c|c|}
\hline Author & Description of study \\
\hline \multicolumn{2}{|l|}{ Cohort studies: } \\
\hline Holmberg et al $1983^{15}$ & $\begin{array}{l}\text { Sweden, two factories. } 13114 \text { workers. Mortaltiy 1961-78. Three exposure groups: }(a) \text { mixers/weighers, }(b) \text { other } \\
\text { production, }(c) \text { white collar. Same factories as Gustavsson et al } 1986 .{ }^{16} \text { Both sexes. }\end{array}$ \\
\hline Gustavsson et al $1986^{16}$ & $\begin{array}{l}\text { Sweden, two factories. } 8374 \text { workers. Mortality } 1952-81 \text {. Cancer incidence } 1959-80 \text {. Minimum employment is one year. } \\
\text { Same factories as Holmberg et al } 1983 .^{15}\end{array}$ \\
\hline Norseth et al $1983^{17}$ & $\begin{array}{l}\text { Norway, } 2448 \text { men employed for } 18 \text { months or more in a footwear and tyre plant between 1953-78. Cancer incidence. } \\
\text { Workers classified in five departments. }\end{array}$ \\
\hline Delzell and Monson $1984^{18}$ & $\begin{array}{l}\text { USA, Akron, Ohio. } 6533 \text { men employed in the industrial products division of a rubber plant (BF Goodrich). Mortality } \\
1940-78 \text {. Same cohort as Delzell and Monson. }{ }^{19-21}\end{array}$ \\
\hline Delzell and Monson $1984^{19}$ & $\begin{array}{l}\text { USA, Akro, Ohio. } 3161 \text { men employed in the aerospace division of a rubber plant (BF Goodrich). Mortality 1940-78. } \\
\text { Same cohort as Delzell and Monson. }{ }^{18} 2021\end{array}$ \\
\hline Delzell and Monson $1985^{20}$ & $\begin{array}{l}\text { USA, Akron, Ohio. } 1152 \text { white men in the tyre-curing department of a rubber plant (BF Goodrich). Mortality 1940-78. } \\
\text { Same cohort as Delzell and Monson. }{ }^{18} 1921\end{array}$ \\
\hline Delzell and Monson $1985^{21}$ & $\begin{array}{l}\text { USA, Akron, Ohio. } 1970 \text { white male reclaim workers of a rubber plant (BF Goodrich). Mortality 1940-78. Same cohort } \\
\text { as Delzell and Monson. }{ }^{18-20}\end{array}$ \\
\hline Wang et al $1984^{22}$ & $\begin{array}{l}\text { China, two tyre factories in Shanghai. Both sexes. Incidence of cancers of the lung, stomach and liver, 1971-80. Workers } \\
\text { classified in five departments. }\end{array}$ \\
\hline Sorahan et al $1986^{23}$ & $\begin{array}{l}\text { UK, BRMA cohort, } 36445 \text { male rubber workers. Mortality 1946-80. Minimum employment is one year. Workers } \\
\text { classified into tyre and general rubber goods sector; by exposure to dusts; by exposure to fumes or solvents. }\end{array}$ \\
\hline Sorahan et al $1989^{24}$ & UK, as in Sorahan et al 1986 . Mortality $1946-85$. \\
\hline Veyes et al $1991^{25}$ & $\begin{array}{l}\text { UK, } 16450 \text { workers. Cancer incidence } 1946-85 \text {. One of the tyre plants included in the BRMA cohort, see also } \\
\text { corrigendum. }{ }^{26}\end{array}$ \\
\hline Bernardinelli et al $1987^{27}$ & Italy, tyre manufacturing factory. 4917 male non-office workers. Mortality 1962-83. \\
\hline Negri et al $1989^{28}$ & Italy, 6629 male workers in rubber tyre factory. Mortality 1946-81. Five job categories. \\
\hline Zhang et al $1989^{29}$ & $\begin{array}{l}\text { China, Shanghai, } 1624 \text { employees in a rubber factory. Both sexes. Mortality from lung cancer } 1972-84 \text {. Classification } \\
\text { of workers by type of job and by exposure to curing agents or talc powder. Adjustment for smoking. }\end{array}$ \\
\hline Szeszenia et al $1991^{30}$ & Poland, 6978 male workers of a rubber goods factory, mainly footwear. Mortality $1945-85$. \\
\hline Carlo et al $1993^{31}$ & $\begin{array}{l}\text { Texas, USA, } 2306 \text { male workers in a tyre manufacturing plant employed for at least one year between } 1962 \text { and } 1989 . \\
\text { Exposure levels reported to have been minimal compared with older cohorts. }\end{array}$ \\
\hline Solionova and Smulevich $1993^{32}$ & $\begin{array}{l}\text { Russia, Moscow, } 1178 \text { men and } 2492 \text { women employed at a rubber footwear and other rubber goods plant for at least } 10 \\
\text { years before } 1983 \text {. Cancer incidence and mortality. Workers classified into production/non-production. }\end{array}$ \\
\hline Weiland et al $1996^{33}$ & Germany, 11663 male rubber workers. Mortality 1981-91. Employment minimum one year, hired between 1910-91. \\
\hline \multicolumn{2}{|l|}{ Studies based on administrative data: } \\
\hline Norell et al $1983^{91}$ & Sweden, record linkage study, 1961-73. Vulcanisation workers. SIR. \\
\hline Malker et al $1987^{92}$ & Sweden, record linkage study, 1961-79. Rubber industry. SIR. \\
\hline Olsen and Jensen $1987^{93}$ & Denmark, record linkage study, $1970-9,76985$ cases. Rubber industry. SPIR. \\
\hline Zheng et al $1992^{94}$ & China, Shanghai, 1980-84. Rubber and plastic workers. SIR. \\
\hline Dolin and Cook-Mozaffari $1992^{95}$ & England and Wales (selected regions), 1965-80. Rubber workers. SIR. \\
\hline Linet et al $1993^{96}$ & Sweden, record linkage study, 1961-79. Rubber manufacturing workers. SIR. \\
\hline Pukkala et al $1995^{97}$ & Finland, record linkage study, 1970-85. Rubber products workers. SIR. \\
\hline Hrubec et al $1995^{98}$ & USA, 1954-80. Rubber workers. Comparative mortality figure (CMF). \\
\hline Costa et al $1995^{99}$ & Italy, Turin, record linkage study, 1981-9. Tyres and rubber production. SMR. \\
\hline
\end{tabular}

SIR = standardised incidence ratio; SPIR = standardised proportional incidence ratio; SMR = standardised mortality ratio.

Replacement of powdered chemicals by chemicals in non-powdered form led to reduced exposures to inhalable particulates in the compounding mixing area. However, $22 \%$ of almost 60 different accelerating, retarding, and antidegrading agents were still being used in powdered form by the end of the 1980s, including several agents classified in either the British Rubber Manufacturers Association B (acute or chronic toxic effects), or Sc (carcinogenic effects) categories. Exposure to solvents was generally low and restricted to workers involved in pretreating. The median fumes concentration in curing departments was as high as $400 \mathrm{mg} / \mathrm{m}^{3}$. Dermal exposure to compounds soluble in cyclohexane was high for workers in engineering services and in compound mixing departments. ${ }^{13}$ When the same companies were revisited five years later in 1994, the chemicals in the Sc category had been either replaced, cut down in use, or used in non-powdered form. ${ }^{14}$

\section{This review}

In this review we summarise the evidence on cancer risks from cohort and case-control studies and from studies with administrative data published after the 1982 IARC evaluation, and examine whether these studies provide more detailed information on the role of specific agents in determining cancer risk. We reviewed studies on the sectors of the rubber industry evaluated by IARC in 1982: tyre manufacturing and repair, cable making, and manufacture of other rubber goods. Studies of workers in the production of natural rubber and synthetic polymers are excluded.

\section{Materials and methods}

Studies were identified through literature searches in commercial data banks (MEDLINE, CANCERLINE, TOXLINE), IARC archives, and in the United States National Cancer Institute computerised reference file. We did not include unpublished data and studies reported only as abstracts. We identified 12 cohort studies from nine countries examining distinct populations of workers in the rubber industry ${ }^{15-33}$; seven nested casecontrol studies in this industry ${ }^{34-40} ; 48$ distinct community based case-control studies from 16 countries that used questionnaire data and reported risks for employment in the rubber industry ${ }^{41-90}$; and finally, 23 studies based on administrative data ${ }^{91-113}$ - such as death certificates, cancer registration, or linkage between census and cancer registry-that presented results by industry or occupation pertinent to assess the risk of cancer among rubber workers. Some of the studies reviewed are updates or more specific investigations of previously reported cohorts. ${ }^{18-21} 232435-40$

All studies are briefly described (table 1 (cohort) and table 2 (case-control)). In some studies, the populations examined partially 


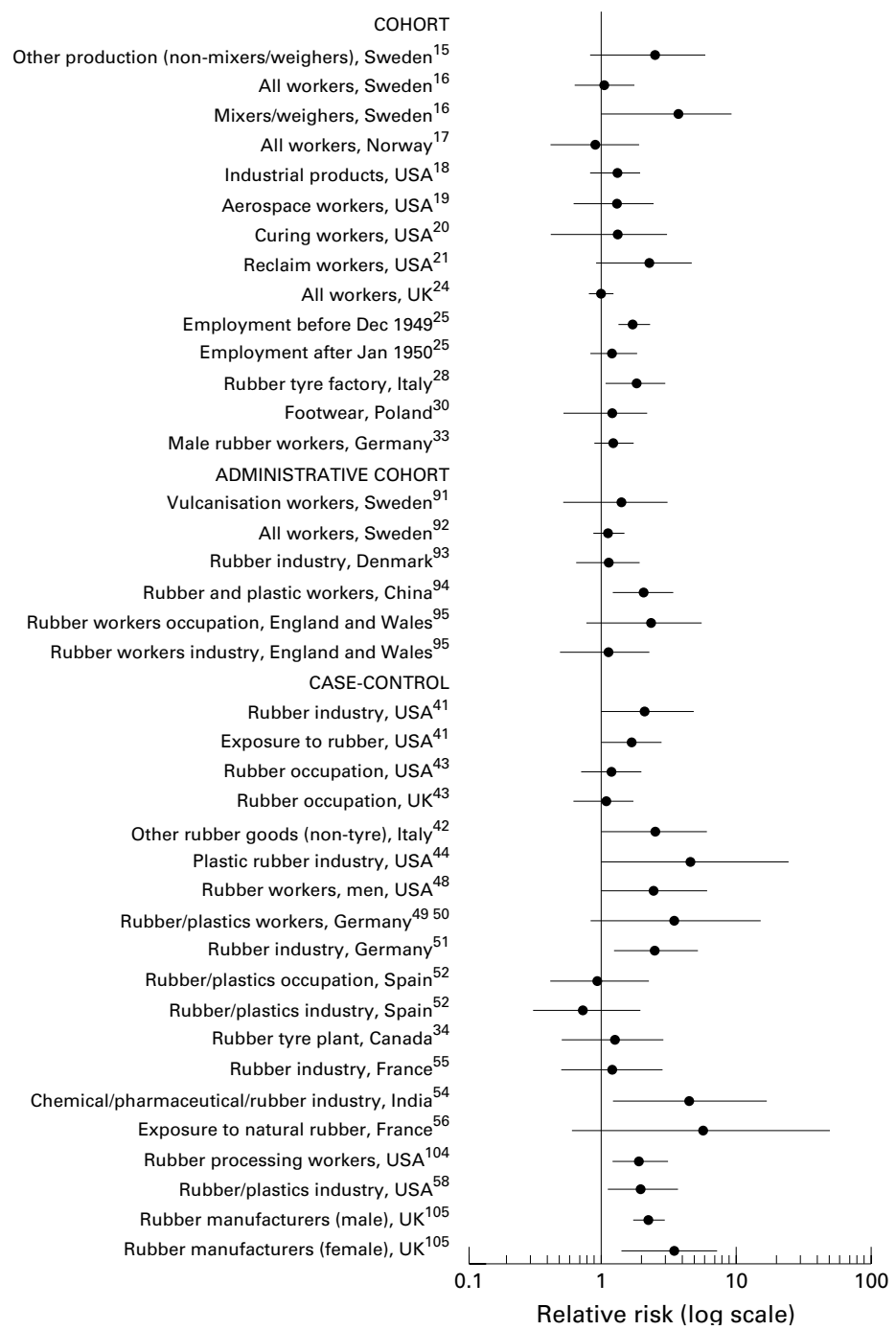

Figure 1 Bladder cancer risk in workers employed in the rubber industry. Odds ratios (95\% CIs) for studies including $>5$ exposed cases.

overlap (tables 1 and 2). Studies are ordered chronologically, grouping subsequent reports of the same study population. We did not further consider in this review, case-control or proportional mortality rate (PMR) studies based on fewer than two exposed cases and controls or those not presenting quantitative risk estimates. ${ }^{47} 536162100$ Studies based on administrative data are clearly noted and are presented with the cohort studies (studies presenting standardised mortality ratios (SMRs), standardised incidence ratios (SIRs), or rate ratios) or with the case-control studies (studies presenting case-control type statistics or PMRs). Studies based on administrative data are often limited in the precision of the measure of exposure which often refers to one point in time (census or death), and, in some cases, in statistical power. Also, preferential reports of positive or significant results may be a problem in these studies. Results from those studies are, therefore, reported only for neoplasms of the bladder, lung, stomach, and lymphatic and haematopoietic tissue. Results for workers in four distinct departments of the BF Goodrich, Akron, Ohio cohort (hereafter noted as the Akron, Ohio cohort), have been published separately ${ }^{18-21}$ and are also reported separately in this review. In the text we make complete reference to all studies included in this review. In the accompanying summary figures (figs 1-5), we have only plotted the results of studies with $>5$ observed cases (cohort studies) or exposed cases (case-control studies), together with those identifying significant results (positive or negative). A detailed report is available from the authors.

\section{Results}

ALL CAUSES OF DEATHS AND ALL MALIGNANT NEOPLASMS

Mortality from all causes was higher than expected based on national reference rates in three cohorts. ${ }^{15} 2433$ Mortality was lower than expected in four studies ${ }^{27} 283031$ and in the four departments of the Akron, Ohio cohort. ${ }^{18-21}$ Cancer incidence or mortality was higher than expected in six cohorts ${ }^{1524-28} 3033$ and in the aerospace, curing, and reclaim departments of the Akron, Ohio cohort. ${ }^{19-21}$ The excesses in SMRs or SIRs were mostly between $5 \%$ and $20 \%$. Cancer incidence or mortality was lower than expected in four cohorts ${ }^{17223136}$ and in the industrial process division of the Akron, Ohio cohort. $^{18}$

\section{BLADDER CANCER}

Figure 1 shows the results on incidence and mortality of bladder cancer.

\section{Cohort studies}

Excess risk of bladder cancer was found in seven distinct studies ${ }^{15-172728303233}$ and in the four departments of the Akron, Ohio cohort, ${ }^{18-21}$ either for the total study population or for workers employed in specific departments. A $>50 \%$ excess risk, ranging from 1.6 to 5.2 , was found in six of these studies and in the reclaim department of the Akron, Ohio cohort. ${ }^{15-17212832}$ No excess risk was found in two studies. ${ }^{24}{ }^{31}$ In four studies reporting results by calendar period, risk was highest among workers employed before the $1950 \mathrm{~s}^{1618} 28{ }^{30}$ who, in one study, were potentially exposed to $\beta$-naphthylamine. ${ }^{30}$ Risk of workers first employed after the 1960s was examined in three studies. ${ }^{273133} \mathrm{~A}$ twofold excess risk was found in the largest study (SMR 2.14, 95\% confidence interval $(95 \% \mathrm{CI}) 1.07$ to $3.84,11$ deaths). ${ }^{33}$ The other two studies had little statistical power to examine bladder cancer risk. Only some studies reported risk in subgroups of workers ${ }^{15-17} 24$ and there is no clear indication of a particular risk in any specific department of the rubber industry, or in workers of specific rubber products. An excess risk was found in five distinct studies based on administrative data ${ }^{91-95}$ but no excess was seen in three. ${ }^{97-99}$

\section{Case-control studies}

Excess risks with odds ratios (ORs) ranging from 1.5 to 5.7 , after adjustment for potential confounding factors such as smoking, were found in 11 case-control studies. 414244464849545658103 Lower excess risks were found in two community based studies ${ }^{43} 55$ and in a nested case-control study ${ }^{34}$ and no excess 
Table 2 Summary data on case-control studies and studies based on administrative data in the rubber industry published after 1982

\begin{tabular}{|c|c|}
\hline Author & Description of study \\
\hline \multicolumn{2}{|l|}{ Bladder cancer: } \\
\hline Schoenberg et al $1984^{41}$ & $\begin{array}{l}\text { USA, New Jersey, } 658 \text { bladder cancer cases; } 1258 \text { population controls. Lifetime occupational history. Partly overlapping } \\
\text { with Zahm et al } 1987 . .^{44}\end{array}$ \\
\hline Coggon et al $1984^{100}$ & $\begin{array}{l}\text { England and Wales, } 291 \text { cases who died of bladder cancer. Two controls per case from all causes of death selected from } \\
\text { death certificate. Usual occupation from death certificates. }\end{array}$ \\
\hline Vineis and Magnani $1985^{42}$ & Italy, Torino, 512 male bladder cancer cases; 596 male hospital controls. Lifetime occupational history. \\
\hline Morrison et al $1985^{43}$ & $\begin{array}{l}\text { US, Boston; UK, Manchester; Japan, Nagoya. 430, 399, and } 226 \text { bladder cancer cases respectively; 397, 493, and } 443 \\
\text { population controls respectively. Occupational history from questionnaire. }\end{array}$ \\
\hline Baxter and McDowall $1986^{101}$ & $\begin{array}{l}\text { England; Six London areas, } 1080 \text { male cases; two controls per case }(a) \text { selected from deaths from all other cancers; }(b) \\
\text { deaths from all causes. Mortality 1968-78. Occupations from death certificate. }\end{array}$ \\
\hline Zham et al $1987^{44}$ & USA, 2982 cases, 5782 population controls. Lifetime occupational history. Partly overlapping with Schoenberg et al $1984{ }^{41}$ \\
\hline Jensen et al $1987^{45}$ & Denmark, Copenhagen, 371 bladder cancer cases; 771 population referents. Lifetime occupational history. \\
\hline Schifflers et al $1987^{46}$ & Belgium, 74 bladder cancer cases; 205 population controls. Lifetime occupational history. \\
\hline Iscovich et al $1987^{47}$ & $\begin{array}{l}\text { Argentina, La Plata, } 117 \text { cases; } 117 \text { hospital and } 117 \text { neighbourhood controls. Three occupations of longest duration and } \\
\text { most recent occupation. }\end{array}$ \\
\hline Risch et al $1988^{48}$ & Canada, 826 bladder cancer cases. 792 population controls. History of occupational exposures and jobs. \\
\hline Claude et al $1989^{49} 50$ & $\begin{array}{l}\text { Germany, } 531 \text { male bladder cancer cases; } 531 \text { hospital controls. Lifetime occupational history. Same study as Kunze et al } \\
1992 . .^{51}\end{array}$ \\
\hline Kunze et al $1992^{51}$ & $\begin{array}{l}\text { Germany, } 675 \text { bladder cancer cases and hospital controls. Lifetime occupational history. Same study as Claude et al } \\
1989 .{ }^{99} 50\end{array}$ \\
\hline Gallagher et al $1989^{102}$ & See entry under multiple cancer sites. \\
\hline Gonzalez et al $1989^{52}$ & Spain, five areas, 497 bladder cancer cases; 530 hospital controls, 530 population controls. Lifetime occupational history. \\
\hline La Vecchia et al $1990^{53}$ & Italy, Milan, 263 bladder cancer cases; 287 hospital controls. Checklist of occupations and exposures. \\
\hline Choi and Nethercott $1991^{34}$ & Canada, proportionate mortality study. 6208 workers in rubber tyre manufacturing plant. Mortality $1917-83$. \\
\hline Hall Rosenman $1991^{103}$ & See entry under multiple tumour sites. \\
\hline Notani et al $1993^{54}$ & See entry under multiple tumour sites. \\
\hline Cordier et al $1993^{55}$ & France, 765 cases; 765 hospital controls. Lifetime occupational history. \\
\hline Hours et al $1994^{56}$ & France, Lyon, 116 cases. Two hospital control groups of 116 subjects each. Lifetime occupational history. \\
\hline Barbone et al $1994^{57}$ & Italy, north-east, 273 male and female cases; 573 hospital controls. Usual occupation. \\
\hline Burnett et al $1994^{104}$ & $\begin{array}{l}\text { USA, } 23 \text { states. } 8644 \text { white male cases who died of bladder cancer. Proportional mortality study. Usual occupation from } \\
\text { death certificates. }\end{array}$ \\
\hline Swanson and Burns $1995^{58}$ & See entry under multiple tumour sites. \\
\hline Roman and Carpenter $1996^{105}$ & UK, England, 1981-7, 16666 cases. Occupation from cancer registry records. Proportional registration ratio. \\
\hline \multicolumn{2}{|r|}{ 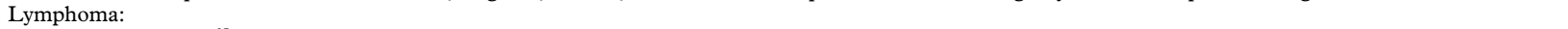 } \\
\hline Wilkosky et al $1984^{35}$ & See entry under multiple tumour sites. \\
\hline Bernard et al $1987^{59}$ & UK, Yorkshire, Hodgkin's disease. 248 cases, 489 hospital controls. Lifetime occupational history. \\
\hline Schumacher and Delzell $1988^{106}$ & $\begin{array}{l}\text { USA, North Carolina, } 501 \text { white and } 1988 \text { black male cases who died of non-Hodgkin's lymphoma; } 569 \text { death certificate } \\
\text { based controls selected from all other deaths. Mortality 1968-70, 1975-77, and 1980-2. Usual occupation and industry } \\
\text { from death certificates. }\end{array}$ \\
\hline Persson et al $1989^{60}$ & $\begin{array}{l}\text { Sweden, Orebro, } 54 \text { cases of Hodgkin's disease; } 106 \text { cases of non-Hodgkin's lymphoma. } 275 \text { population referents. } \\
\text { Checklist of occupational exposures. }\end{array}$ \\
\hline La Vecchia et al $1989^{61}$ & $\begin{array}{l}\text { Italy, Milan, both sexes. Hodgkin's disease, } 69 \text { cases; non-Hodgkin's lymphomas, } 153 \text { cases; } 396 \text { hospital controls } \\
\text { frequently matched. Checklist of occupations and exposures. }\end{array}$ \\
\hline Francheschi et al $1991^{62}$ & Italy, north-east, Pordenone province. 152 cases; 613 hospital controls. Checklist of occupations and exposures. \\
\hline Blair et al $1993^{63}$ & $\begin{array}{l}\text { USA, Iowa and Minnesota, } 622 \text { white non-Hodgkin's lymphoma male cases; } 1245 \text { population based white male controls. } \\
\text { Lifetime occupational history. }\end{array}$ \\
\hline \multicolumn{2}{|r|}{ 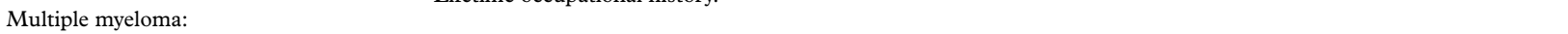 } \\
\hline La Vecchia et al $1989^{61}$ & Italy, Milan, 110 cases; 396 frequency matched hospital controls. Checklist of occupations and exposures. \\
\hline Heineman et al $1992^{107}$ & $\begin{array}{l}\text { Denmark, } 1098 \text { men diagnosed between } 1970-84 ; 4169 \text { controls chosen randomly from the Danish central population } \\
\text { registry. Lifetime occupational history from pension fund records. }\end{array}$ \\
\hline Pottern et al $1992^{64}$ & $\begin{array}{l}\text { Denmark, } 363 \text { women diagnosed between } 1970-84 ; 1517 \text { controls selected from the central population registry. Lifetime } \\
\text { occupational history from pension fund records. }\end{array}$ \\
\hline Demers et al $1993^{65}$ & $\begin{array}{l}\text { USA counties in Washington State; metropolitan Atlanta; metropolitan Detroit, } 693 \text { cases diagnosed between 1977-81; } \\
1683 \text { population based controls. Lifetime job history. }\end{array}$ \\
\hline Figgs et al $1994^{108}$ & $\begin{array}{l}\text { USA, } 24 \text { states. } 12148 \text { male and female deceased subjects cases; } 60740 \text { controls dying from diseases other than cancer, } \\
\text { selected from the death certificate data base. Mortality } 1984-9 \text {. Occupation and industry from death certificate. }\end{array}$ \\
\hline \multicolumn{2}{|r|}{ os } \\
\hline Arp, Jr et al $1983^{36}$ & $\begin{array}{l}\text { USA, nested case-control study within a cohort of rubber industry workers (McMichael et al } 1975){ }^{12} 15 \text { male and } \\
\text { female cases of lymphocytic leukaemia; } 30 \text { matched industry controls. Mortality } 1964-73 \text {. Historical solvent exposure } \\
\text { profile for rubber industry job titles. }\end{array}$ \\
\hline Wilkosky et al $1984^{35}$ & See entry under multiple tumour sites. \\
\hline Dubrow and Wegman $1984^{109}$ & See entry under multiple tumour sites. \\
\hline Flodin et al $1987^{61}$ & $\begin{array}{l}\text { Sweden, middle and south east, } 111 \text { white men and women diagnosed with chronic lymphatic leukaemia; } 431 \\
\text { randomised population referents. Checklist of occupational exposures. }\end{array}$ \\
\hline Malone et al $1989^{67}$ & $\begin{array}{l}\text { USA; western Washington State; northern Utah; Detroit, Michigan; Atlanta, Georgia, } 427 \text { men and women diagnosed } \\
\text { with chronic lymphocytic leukaemia } 1977-81 ; 1683 \text { population based controls. History of chemical exposure and } \\
\text { employment of six months or more in four specific industries. }\end{array}$ \\
\hline Gallagher et al $1989^{102}$ & See entry under multiple cancer sites. \\
\hline Loomis and Savitz $1991^{110}$ & $\begin{array}{l}\text { USA; } 16 \text { states, } 5147 \text { male deceased subjects with leukaemia; } 51470 \text { controls (other causes of death). Subjects from } \\
1985-7 \text { mortality data tapes from the National Centre for Health Statistics (NCHS). Occupation from death }\end{array}$ \\
\hline & certificate. \\
\hline \multicolumn{2}{|l|}{ Lung cancer: } \\
\hline Delzell et al $1982^{37}$ & $\begin{array}{l}\text { USA, Akron, Ohio (University of N Carolina cohort, McMichael et al 1975), }{ }^{12} \text { nested case-control study. } 121 \text { white male } \\
\text { cases; } 448 \text { controls who died of other causes. Mortality } 1964-73 \text {. Detailed employment history in } 19 \text { work areas. }\end{array}$ \\
\hline Milne et al $1983^{111}$ & $\begin{array}{l}\text { USA; California, Alameda County, } 925 \text { lung cancer deaths; } 6420 \text { deaths with other cancers identified through death } \\
\text { certificates. White and non-white males and females. Mortality 1958-62. Usual occupation and industry from death } \\
\text { certificate. }\end{array}$ \\
\hline Dubrow and Wegman $1984^{109}$ & See entry under multiple tumour sites. \\
\hline Wilkosky et al $1984^{35}$ & See entry under multiple tumour sites. \\
\hline Coggon et al $1984^{100}$ & $\begin{array}{l}\text { UK, England and Wales, } 598 \text { cases who died of lung cancer. Two controls per case from all other deaths selected from } \\
\text { death certificate. Usual occupation from death certificate. }\end{array}$ \\
\hline Kjuus et al $1986^{68}$ & Norway, southeast, 176 male cases and 176 hospital controls. Main lifetime occupations or exposures. \\
\hline Schoenberg et al $1987^{69}$ & $\begin{array}{l}\text { USA, six areas of New Jersey, } 763 \text { white male incident cases; } 900 \text { general population controls. Lifetime occupational } \\
\text { history. }\end{array}$ \\
\hline Andjelkovich et al $1988^{38}$ & $\begin{array}{l}\text { USA, University of North Carolina, nested case control study. } 40 \text { lung cancer cases occurring 1977-8; } 148 \text { controls. } 20 \\
\text { occupational title groups. }\end{array}$ \\
\hline Levin et al $1988^{70}$ & China, Shanghai, 733 male cases; 760 population controls. Lifetime occupational history. \\
\hline
\end{tabular}


Table 2 continued

\begin{tabular}{|c|c|}
\hline Author & Description of study \\
\hline Gallagher et al $1989^{102}$ & See entry under multiple tumour sites. \\
\hline Burns and Swanson $1991^{71}$ & $\begin{array}{l}\text { USA, Michigan, Detroit metropolitan area, } 5935 \text { male and female cases; } 3956 \text { incident colon and rectum cancer } \\
\text { referents. Complete occupational history. }\end{array}$ \\
\hline Jockel et al $1992^{72}$ & $\begin{array}{l}\text { Germany, Bremen, Hamburg, Hannover, Cologne, and Bielefeld, } 194 \text { incident male and female cases; } 194 \text { hospital and } \\
194 \text { population controls. Lifetime history of occupation and occupational exposures. }\end{array}$ \\
\hline Brownson et al $1993^{73}$ & $\begin{array}{l}\text { USA, Missouri. } 429 \text { white female incident cases aged } 30 \text { to } 84 \text { years; } 1021 \text { population based female controls. Checklist } \\
\text { of selected occupations, industries and occupational exposures. }\end{array}$ \\
\hline Notani et al $1993^{54}$ & See entry under multiple tumour sites. \\
\hline Wu-Williams et al $1993^{74}$ & China, Shenyang and Harbin, 965 female cases; 959 population controls. Lifetime occupational history. \\
\hline Swanson and Burns $1995^{58}$ & See entry under multiple tumour sites. \\
\hline Wang et al $1995^{112}$ & $\begin{array}{l}\text { China, Tianjin, cancer registry based case-control study. } 8401 \text { male and female lung cancer cases; } 27695 \text { cancer control. } \\
\text { Current or more recent occupation at the time of registration. }\end{array}$ \\
\hline \multicolumn{2}{|r|}{ 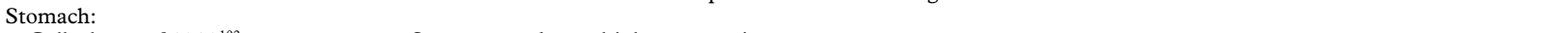 } \\
\hline Gallagher et al $1989^{102}$ & See entry under multiple tumour sites. \\
\hline Coggon et al $1990^{75}$ & UK, 95 male and female cases; 190 community controls. Lifetime occupational histories. \\
\hline Gonzalez et al $1991^{76}$ & $\begin{array}{l}\text { Spain, four areas, } 354 \text { histologically confirmed male and female cases; } 354 \text { hospital referents. Lifetime occupational } \\
\text { history. }\end{array}$ \\
\hline Cocco et al $1994^{77}$ & Italy, six areas, 640 histologically confirmed male cases; 959 population controls. Three longest held jobs. \\
\hline Ward et al $1994^{113}$ & $\begin{array}{l}\text { USA, } 24 \text { states, white women deceased from cancer of the gastric cardia }(n=128) \text {, cardia/lower oesophagus }(n=162) \text { and } \\
\text { other gastric }(n=6065) \text {; five controls per case randomly selected from deaths from other causes except cancer and } \\
\text { gastrointestinal disorders. Mortality } 1984-89 \text {. Occupation/industry from death certificate. }\end{array}$ \\
\hline \multicolumn{2}{|l|}{ Colon: } \\
\hline Peters et al $1989^{78}$ & $\begin{array}{l}\text { USA, Los Angeles County, } 147 \text { white men cases ( } 72 \% \text { colon and } 28 \% \text { rectal cancer); individually matched } \\
\text { neighbourhood controls. Lifetime occupational history. }\end{array}$ \\
\hline Swanson and Burns $1995^{58}$ & See entry under multiple tumour sites. \\
\hline \multicolumn{2}{|l|}{ Liver: } \\
\hline Swanson and Burns $1995^{58}$ & See entry under multiple tumour sites. \\
\hline \multicolumn{2}{|l|}{ Larynx: } \\
\hline Zagraniski et al $1986^{79}$ & $\begin{array}{l}\text { USA, Connecticut, New Haven, } 92 \text { white male cases diagnosed between 1975-80; } 181 \text { hospital controls. Lifetime } \\
\text { occupational history. }\end{array}$ \\
\hline Muscat et al $1991^{80}$ & $\begin{array}{l}\text { USA, New York, Illinois, Michigan, and Pennsylvania, } 194 \text { white male cases; } 184 \text { age matched hospital controls. Six } \\
\text { longest held occupations. }\end{array}$ \\
\hline \multicolumn{2}{|l|}{ Skin: } \\
\hline Bourguet et al $1989^{39}$ & $\begin{array}{l}\text { USA, Akron, Ohio, } 65 \text { white male rubber workers with squamous cell carcinoma of the skin between } 1964-81 ; 254 \\
\text { matched controls, workers in four major American rubber manufacturing companies. Lifetime occupational history. }\end{array}$ \\
\hline \multicolumn{2}{|r|}{ 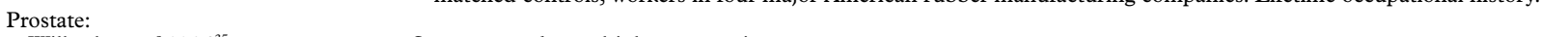 } \\
\hline Wilkosky et al $1984^{35}$ & See entry under multiple tumour sites. \\
\hline Elghany et al $1990^{81}$ & USA, Utah, 358 cases; 679 population controls. Longest and most recent industry/occupation. \\
\hline Le Marchand et al $1991^{82}$ & USA, Hawaii, Oahu, 452 cases; 898 population controls. Lifetime occupational history. \\
\hline \multicolumn{2}{|l|}{ Kidney and renal pelvis: } \\
\hline McLaughlin et al $1987^{83}$ & $\begin{array}{l}\text { USA, Minneapolis, St Paul metropolitan area, } 74 \text { white male and female cases diagnosed between 1974-9; } 697 \\
\text { population controls. Usual occupation and checklist of exposures. }\end{array}$ \\
\hline Partanen et al $1991^{84}$ & $\begin{array}{l}\text { Finland, } 338 \text { white men and women diagnosed between 1977-8; } 484 \text { matched population referents. Lifetime } \\
\text { occupational history. }\end{array}$ \\
\hline \multicolumn{2}{|r|}{ - } \\
\hline Symons et al $1982^{40}$ & $\begin{array}{l}\text { USA, Ohio, Akron. Nested case control study in a plant of manufacturing rubber products, University of N Carolina } \\
\left.\text { cohort (Andjelkovic et al } 1976,{ }^{9} \mathrm{McMichael} \text { et al } 1976^{10}\right) .22 \text { brain and central nervous system cases. Controls from the } \\
\text { plant. Mortality 1952-71. Complete work history. }\end{array}$ \\
\hline Burch et al $1987^{85}$ & $\begin{array}{l}\text { Canada, southern Ontario, } 215 \text { men and women diagnosed with brain tumour between } 1979-82 ; 215 \text { hospital controls. } \\
\text { History of occupations. }\end{array}$ \\
\hline Preston-Martin et al $1989^{86}$ & $\begin{array}{l}\text { USA, California, Los Angeles County, } 272 \text { black and white men with a primary brain tumour first diagnosed during } \\
1980-4 ; 272 \text { matched neighbour controls. Detailed occupational histories. }\end{array}$ \\
\hline \multicolumn{2}{|r|}{ 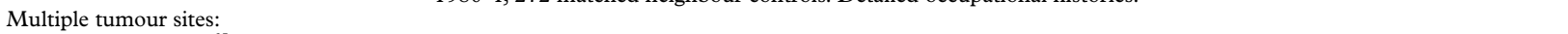 } \\
\hline Wilkosky et al $1984^{35}$ & $\begin{array}{l}\text { USA, nested case-control study within } 6678 \text { cohort members of rubber and tyre manufacturing plant, Akron, Ohio } \\
\text { (University of North Carolina cohort, McMichael et al } 1974^{8} \text { ). Cases of lung, stomach, prostate, lymphatic, and } \\
\text { haematopoietic neoplasms dying between } 1964-73 \text {. Controls, } 20 \% \text { random sample of cohort. Exposure to } 20 \text { specific } \\
\text { solvents. }\end{array}$ \\
\hline Hall and Rosenman $1991^{103}$ & $\begin{array}{l}\text { USA, New Jersey state cancer registry, 1979-84. Proportional cancer incidence study. Results on rubber production } \\
\text { reported only for bladder, liver, uterine cancer, and melanoma out of } 38 \text { distinct cancer sites examined. Occupational } \\
\text { history from registration record. }\end{array}$ \\
\hline Notani et al $1993^{54}$ & $\begin{array}{l}\text { India, Bombay, male cases of lung cancer }(n=246) \text { and bladder cancer }(n=153) ; 212 \text { male cancer controls. Lifetime } \\
\text { occupational history. }\end{array}$ \\
\hline Swanson and Burns $1995^{58}$ & $\begin{array}{l}\text { USA, Michigan, Detroit, women. } 2015 \text { lung cancer cases; } 1558 \text { colon cancer; } 627 \text { bladder cancer, } 157 \text { liver cancer. } 1972 \\
\text { population controls. Lifetime occupational history. Same cases as Burns and Swanson } 1991 .\end{array}$ \\
\hline Dubrow and Wegman $1984^{109}$ & $\begin{array}{l}\text { USA, Massachusetts, } 34879 \text { deaths, 1971-3. Rubber workers. Standardised mortality odds ratio. Occupational history } \\
\text { from death certificates. }\end{array}$ \\
\hline Gallagher et al $1989^{102}$ & Canada, BC, 1950-84. 320423 deaths. Proportional mortality ratio. Occupational exposure from death certificates. \\
\hline
\end{tabular}

SIR = standardised incidence ratio; SPIR = standardised proportional incidence ratio; SMR = standardised mortality ratio.

in three. ${ }^{45} 5257$ Exposure groups in most casecontrol studies were defined on the basis of lifetime occupational history and referred to employment in the rubber industry. In some studies, the exposed group included workers employed in the rubber and plastics industry, ${ }^{52} 445849103$ or in even wider industrial areas. $^{465}$ In the study by Cordier et $a l,{ }^{55}$ no excess risk was found among workers employed during the period of use of $\beta$-naphthylamine. Two studies based on administrative data, in England and the United States, found an excess risk $^{104105}$; one study found no excess risk. ${ }^{101}$
NEOPLASMS OF THE LYMPHATIC AND HAEMATOPOIETIC TISSUE

Figure 2 shows the results on neoplasms of the lymphatic and haematopoietic tissue.

\section{Malignant lymphomas}

Cohort studies-Excess risks ranging from 1.7 to 3.6 were found in two cohort studies in Italy (Hodgkin's disease only) ${ }^{27}$ and Norway ${ }^{17}$ and in the industrial products and aerospace departments of the Akron, Ohio cohort. ${ }^{18}{ }^{19}$ Excess risks were found in various jobs or departments. No excess risk was found in two cohort 


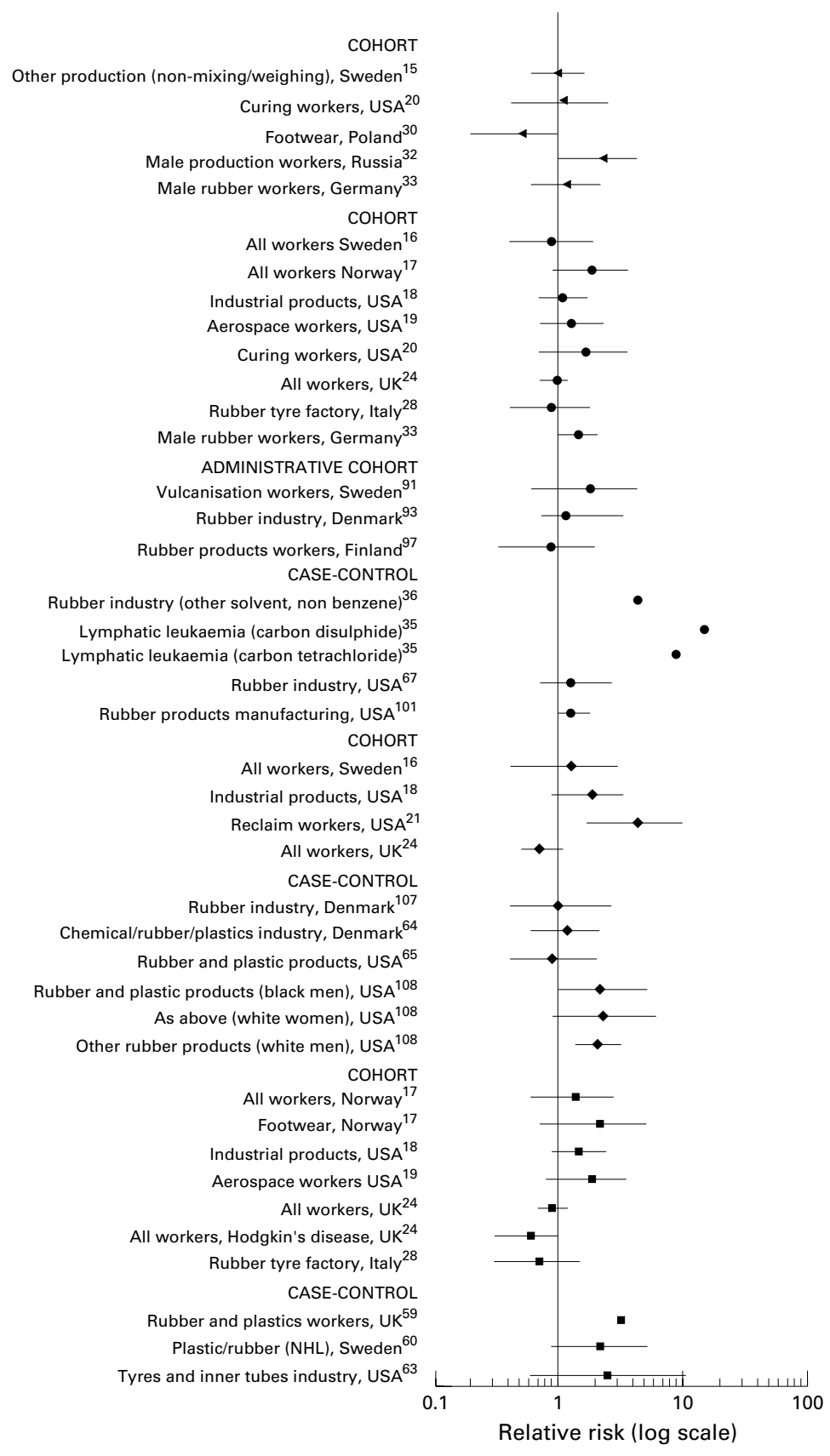

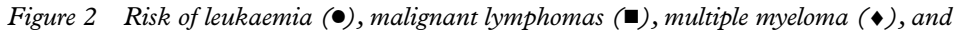
unspecified neoplasms of the lymphatic and haematopoietic tissue $(\mathbf{\Lambda})$ in workers employed in the rubber industry. Odds ratios (95\% CIs) for studies including $>5$ exposed cases.

studies $^{24}{ }^{28}$ and in one study based on administrative data. ${ }^{96}$

Case-control studies-Excess risk of nonHodgkin's lymphoma was found in two casecontrol studies among workers employed in the tyre and inner tubes industry ${ }^{63}$ or in the rubber and plastics industry. ${ }^{60}$ Excess risk for Hodgkin's disease was found in one study ${ }^{59}$ but no excess risk was found in another. ${ }^{60}$ An excess risk was also found in one study that used administrative data. ${ }^{106}$

\section{Multiple myeloma}

Cohort studies - Excess risks ranging from 1.3 to 4.3 were found in one Swedish study ${ }^{16}$ and in the industrial products and reclaim depart- ments of the Akron, Ohio cohort. ${ }^{18}{ }^{21}$ No excess was found in one study. ${ }^{24}$

Case-control studies-No excess risk was found in one study ${ }^{65} \mathrm{~A}$ twofold excess risk was found among men and women in three studies that used administrative data. ${ }^{64107} 108$

\section{Leukaemia}

Cohort studies-Moderately increased leukaemia risks ranging from 1.5 to 2.3 were found in three cohort studies, ${ }^{17} 2733$ and in the tyre curing department of the Akron, Ohio cohort in the United States. ${ }^{20}$ Lower excess risks were seen in the industrial products and aerospace departments of the Akron, Ohio cohort. ${ }^{18}{ }^{19}$ No excess risk was found in four cohort studies $^{16242831}$ and in the reclaim department of the Akron, Ohio cohort. ${ }^{21}$ The risk of leukaemia was not centred in specific departments, and in most studies was not examined in relation to exposure to specific agents. In the Norwegian cohort, ${ }^{17}$ six out of nine leukaemia cases had documented exposure to glues with a concentration of up to $4 \%$ benzene. An excess risk, ranging from 1.2 to 2.9 , was found in three studies based on administrative data, ${ }^{91}{ }^{93}{ }^{99}$ but no excess was found in two. ${ }^{97} 98$ Case-control studies-High excess risks associated with exposure to benzene ${ }^{36}$ or other solvents ${ }^{35} 36$ were found in two nested casecontrol studies within rubber industry cohorts. Excess risks lower than 1.5 were found in two case-control studies. ${ }^{66}{ }^{67}$ An excess risk was found in three studies based on administrative data. ${ }^{102} 109110$

\section{Unspecified lymphatic and haematopoietic neoplasms}

Results were presented jointly for lymphoma and leukaemia in three cohort studies. ${ }^{15} 3032 \mathrm{~A}$ twofold excess risk was found in the study from Russia. $^{32}$ Results were presented jointly for lymphomas and multiple myeloma in two cohort studies, in which no excess risk was found. ${ }^{3133}$

LUNG CANCER

Figure 3 shows the results on lung cancer.

\section{Cohort studies}

Excess risks (ranging from 1.7 to 3.3) were found in four cohort studies ${ }^{15} 16293032$ and in the curing department of the Akron, Ohio cohort. ${ }^{20}$ These risks were found in a variety of departments including workers in tyre curing departments, mixing and milling, vulcanisation workers, and in one study in jobs with high exposure fumes or solvents. Excess risks $<1.5$ were found in two studies ${ }^{24} 33$ and in the aerospace department of the Akron, Ohio cohort. $^{19}$ No excesses were found in the remaining four cohort studies ${ }^{17} 272831$ and in the industrial products and reclaim departments of the Akron, Ohio cohort. ${ }^{18}{ }^{21}$ In two cohorts, risk was highest among workers employed before the 1950 s. $^{1620}$ In studies including workers first employed after 1960, no excess was found in two studies, ${ }^{27} 31$ whereas a small excess (SMR 1.36, 95\% CI 1.04 to 1.75 , 60 deaths) was found in the German study. ${ }^{33}$ 


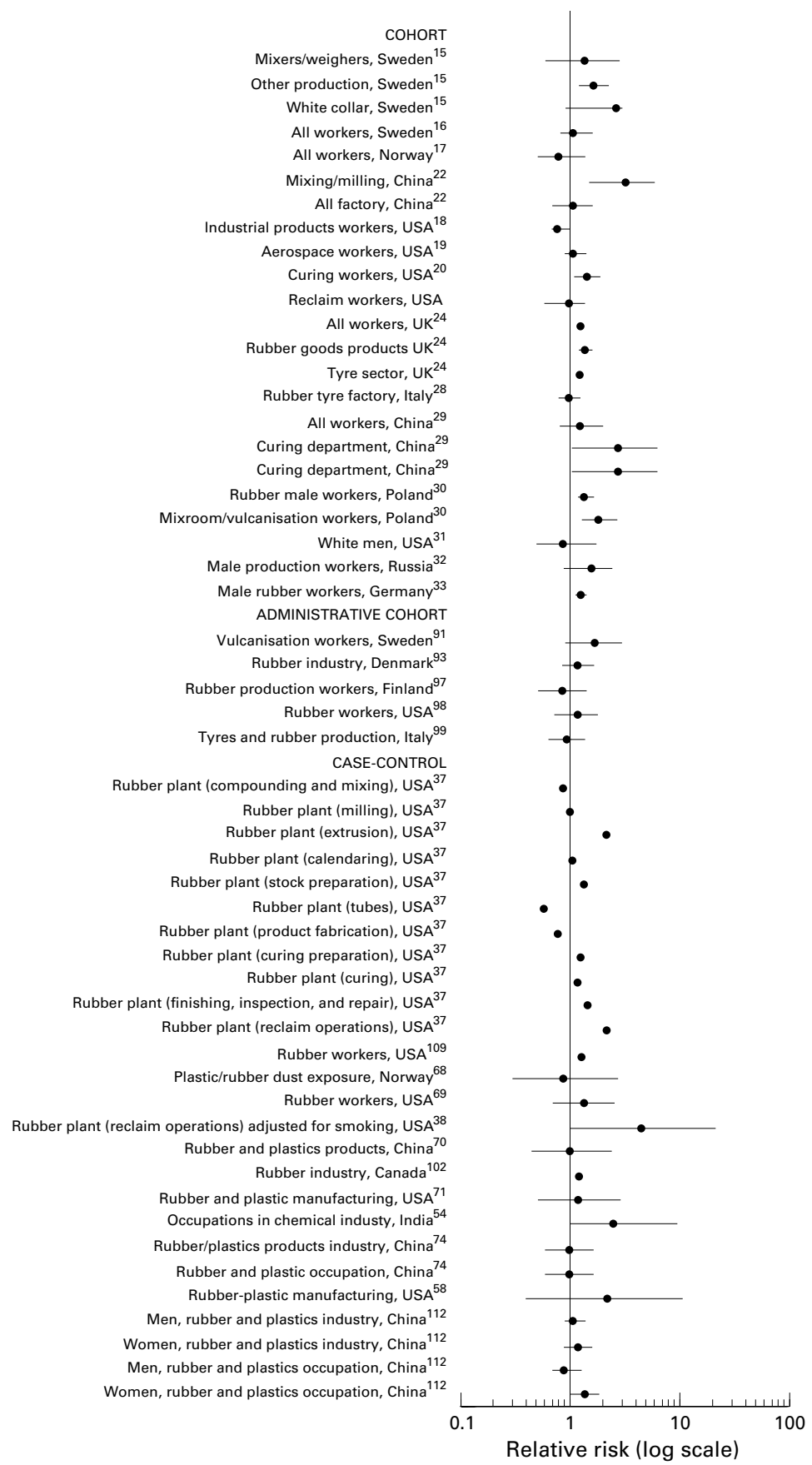

Figure 3 Lung cancer risk in workers employed in the rubber industry.

The smoking habits of a subgroup of workers were examined in one study ${ }^{29}$ and the findings suggested that the excess risk of lung cancer could not be attributed to smoking. Risks ranging form 1.2 to 1.75 were found in three administrative data studies, ${ }^{91} 9398$ whereas no excesses were found in two record linkage studies. ${ }^{97} 99$

Case-control studies

Excess risks ranging from 1.5 to 4.6 were found in three community based studies ${ }^{54} 5873$ and in two nested case-control studies. ${ }^{37} 38$ Lower excess risks were found in two studies, ${ }^{69} 71$ and no excess risks were found in four community based studies. ${ }^{687072} 74$ No association was found with exposure to solvents in a nested case- control study. ${ }^{35}$ In seven studies ${ }^{54} 586870-7274$ the exposed group included workers in the rubber and plastics industry. An excess risk was found in four studies based on administrative data. ${ }^{102109111112}$

STOMACH CANCER

Figure 4 shows the results on stomach cancer.

Cohort studies

Low excess risks not exceeding 1.6 were found in seven studies. ${ }^{162224273233}$ The risk was concentrated in the mixing and milling departments in two studies, ${ }^{162}$ and in jobs with high exposure to dust in the British Rubber Manufacturers Association, United Kingdom cohort. ${ }^{24}$ No excesses were found in four studies $^{17} 283031$ and in the industrial products, curing, and reclaim departments of the Akron, Ohio cohort. ${ }^{1820}$ In none of the four studies based on administrative data that reported results for stomach cancer was the risk $>5 \%$. ${ }^{91} 939899$

\section{Case-control studies}

Risk of stomach cancer was associated with long term manual employment in the rubber industry in an English study (OR 3.5), ${ }^{75}$ and with exposure to rubber or talc in a Spanish study (OR 1.65). ${ }^{76} \mathrm{~A}$ lower excess risk was found in one study which examined exposure of workers in the rubber or chemical industries. ${ }^{77}$ In one study based on administrative data the risk was not $>5 \%,{ }^{102}$ and in another study the risk depended on the position of the tumour. ${ }^{113}$

\section{CANCER OF THE OESOPHAGUS}

\section{Cohort studies}

An increased risk (SMR 2.7) of neoplasms of the oesophagus was found among reclaim workers in the Akron, Ohio cohort. ${ }^{21}$ Lower risks, not $>1.5$, were found in three cohort studies, ${ }^{24} 3033$ the excess being significant in one study $^{24}$ (SMR 1.2, 95\% CI 1.0 to 1.5$)$. No excess risks were found in three other cohort studies $^{162832}$ and in the industrial process division of the Akron, Ohio cohort. ${ }^{18}$

\section{COLON CANCER}

\section{Cohort studies}

A low excess risk was found in three studies $^{173032}$ and in the curing department of the Akron, Ohio cohort. ${ }^{20}$ No excess risks were found in three other studies, ${ }^{162433}$ and in the reclaim department of the Akron, Ohio study. ${ }^{21}$

\section{Case-control studies}

A threefold excess risk for rubber and plastic workers was found in one ${ }^{58}$ out of two available studies. $^{78}$

\section{CANCER OF THE PANCREAS \\ Cohort studies}

Excess risks of cancer of the pancreas ranging from 1.5 to 2.6, were found in four studies, ${ }^{15}{ }^{30-32}$ and in the tyre curing department of the Akron, Ohio plant. ${ }^{20}$ Lower excess risks were found in one additional study ${ }^{17}$ and in the reclaim department of the Akron, Ohio 


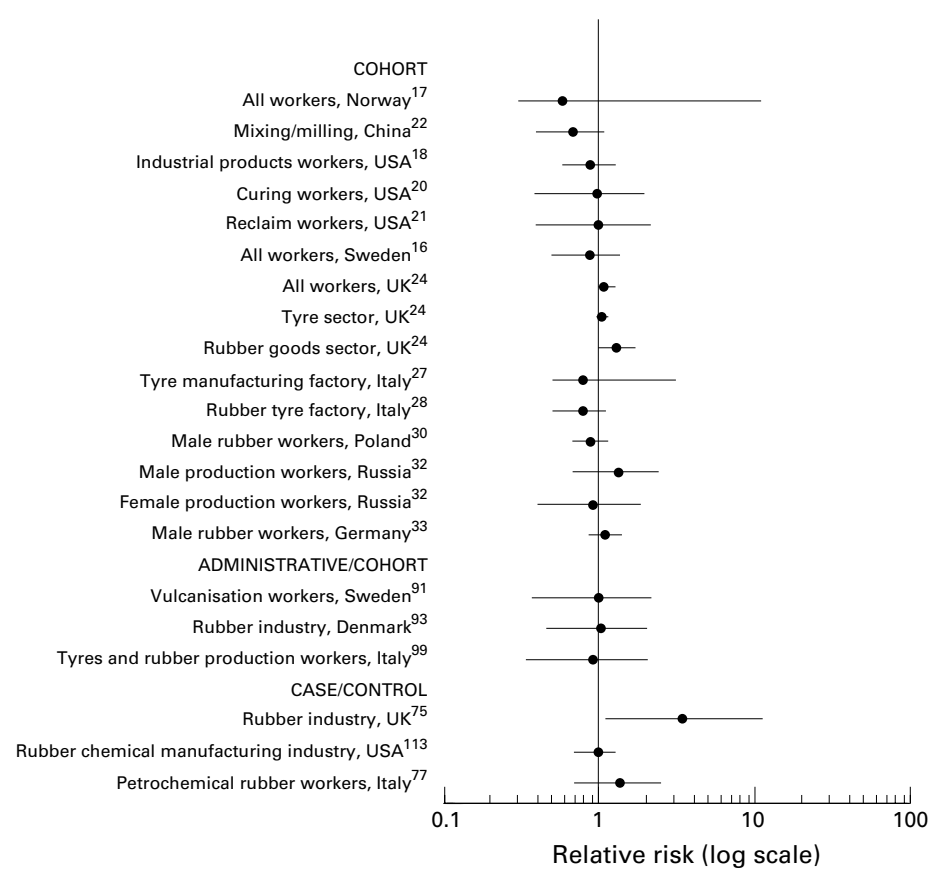

Figure 4 Stomach cancer risk in workers employed in the rubber industry. Odds ratios ( $95 \%$ CIs) for studies including $>5$ exposed cases.

cohort. ${ }^{21}$ No excess risk was found in four cohort studies $^{24-28} 33$ and in the industrial products department of the Akron, Ohio cohort. ${ }^{18}$

\section{LIVER CANCER}

Cohort studies

A higher than fourfold risk for liver cancer was found in two studies. ${ }^{15} 27 \mathrm{~A}$ lower excess risk was found in the BRMA cohort in the United Kingdom (SMR 1.4), ${ }^{24}$ whereas no excess was found in four other studies. ${ }^{22} 283033$

\section{Case-control studies}

A twofold excess risk for subjects employed in rubber or plastics manufacturing, was found in a population based case-control study in the United States. ${ }^{58}$

CANCER OF THE LARYNX

Figure 5 shows the results of the studies on laryngeal cancer.

\section{Cohort studies}

Low or moderate excess risks were found in all seven studies reporting results on laryngeal cancer, ${ }^{1624-28303233}$ although $95 \%$ CIs were

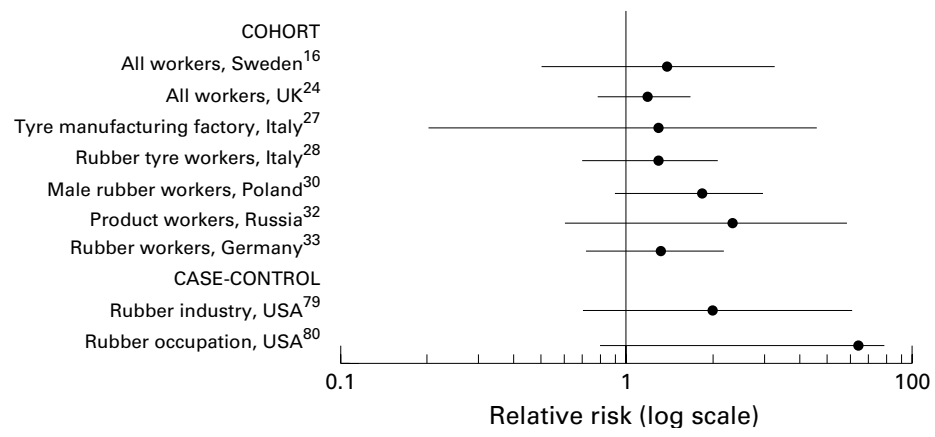

Figure 5 Laryngeal cancer risk in workers employed in the rubber industry. Odds ratios ( $95 \%$ CIs) for studies including $>5$ exposed cases. wide. The highest risks were found for workers in Russia ${ }^{32}$ and Poland. ${ }^{30}$

Case-control studies

Two case-control studies in the United States found a high risk among rubber industry workers. ${ }^{79} 80$

NON-MELANOCYTIC SKIN CANCER

Cohort studies

An excess risk was found in three studies ${ }^{15}{ }^{27} 28$ but no excess was seen in the remaining four cohort studies that reported results for this cancer. ${ }^{24} 303233$

Case-control studies

Excess risks among workers exposed to rubber stock (OR 2.2) and lubricating oils (OR 6.5) were found in a nested case-control study in rubber industries in Akron Ohio. ${ }^{39}$

BRAIN TUMOURS

Cohort studies

An excess risk was found in five studies. ${ }^{15} 1727303234$ No excess risk was found in four other studies. ${ }^{2428} 3133$

\section{Case-control studies}

A higher than twofold excess risk was found in two studies in Canada ${ }^{85}$ (OR 9.0), and the United States $^{86}$ (OR 3.5). No excess (OR 0.4) was found in a nested case-control study in the Akron, Ohio cohort. ${ }^{40}$

\section{PROSTATE CANCER}

Cohort studies

An excess risk was found in four studies ${ }^{17} 273233$ and in the industrial products department of the Akron, Ohio cohort. ${ }^{18}$ No excess risks were found in three other studies ${ }^{1624} 30$ and in the aerospace and reclaim departments of the Akron, Ohio cohort. ${ }^{1921}$

\section{Case-control studies}

No excess risk was found in two case control studies that reported risk for employment in the rubber industry. ${ }^{81} 82$

\section{KIDNEY CANCER}

Cohort studies

A low excess risk not exceeding 1.5 was found in three studies, ${ }^{28} 3032$ and in the reclaim department of the Akron, Ohio cohort. ${ }^{21}$ No excess was found in two cohort studies, ${ }^{16}{ }^{24}$ and in the industrial products department of the Akron, Ohio cohort. ${ }^{18}$

Case-control studies

A higher than twofold risk was found in two studies. ${ }^{83} 84$

\section{OTHER CANCERS}

An excess risk of thyroid cancer was found in the BRMA cohort (SMR 1.7, 9 deaths) ${ }^{24}$ and in an Italian cohort study (SMR 5.0, 1 death $)^{27}$; no excess was found in a study in Russia. ${ }^{32}$ Excess risks have been described sporadically both in cohort and in case-control studies for other neoplasms such as cancers of the 
nasopharynx, ${ }^{88}$ the oropharynx, ${ }^{89} 97$ and the uterus, ${ }^{103}$ malignant melanoma, ${ }^{103}$ and soft tissue sarcoma. ${ }^{90}$

\section{Discussion}

A moderate excess risk of cancer of the bladder and the lung and of leukaemia was found in most studies of workers employed in the rubber industry in different regions of the world. A small but consistent excess risk was found for laryngeal cancer. There was an indication of an excess risk of other neoplasmssuch as lymphomas and pancreatic and brain tumours-but results were not consistent between studies. There was little evidence of an excess risk of stomach cancer. Detailed exposure information was lacking in nearly all available studies and an evaluation of cancer risk by specific exposures and processes within the rubber industry was, therefore, not possible.

We based our conclusions on the consistency of results across studies and on the magnitude of the reported risks. We did not pay much attention to statistical significance, nor did we attempt to apply techniques of meta-analysis and calculate pooled risk estimators. ${ }^{114}$ Firstly, many studies were small and although they identified excess risks, they had little power to detect significant results. For example, in very few studies was the excess risk of leukaemia significant at the conventional $\alpha$ level of 0.05 . It is possible, however, that the excess risk of leukaemia is causally related to occupational exposures in this industry, given the recorded widespread exposure to solvents in the past. By contrast, small effects- such as the $10 \%-20 \%$ excess risks for oesophageal and stomach cancer, which in the large BRMA cohort in the United Kingdom were significant - could have resulted from confounding by socioeconomic status. Secondly, the heterogeneity of exposure circumstances within and between plants, ${ }^{13}$ differences of occupational and exposure classifications used in the studies, and differences in study design, complicates the calculation of pooled estimators for specific exposures or departments, and makes the calculation of a single summary risk estimate uninformative. Finally, although nearly all cohort studies presented results for cancers of the bladder, lung, stomach, pancreas, and leukaemia and lymphomas, reporting was incomplete for other cancers. Reporting for other sites may have been biased with positive findings overreported even when based on small numbers. A similar argument may hold true for case-control studies and the reporting of risks for employment in the rubber industry.

A moderate excess risk of bladder cancer was found in most of the new studies. This excess risk had been associated in earlier studies with exposure to an antioxidant contaminated with $\beta$-naphthylamine, ${ }^{1}$ and removal of this agent from rubber plants in the United Kingdom has been shown to prevent the occurrence of further cases of bladder cancer. ${ }^{5}$ However, in many recent studies conducted in both developed and developing countries, an excess cancer risk was found among workers with no recorded exposure to $\beta$-naphthylamine. This indicates that other agents in this industry may also be associated with the occurrence of bladder cancer among rubber workers. This finding implies that the removal of a single agent from this industry, although important for diminishing the risk in some plants, may not suffice to eliminate the excess risk of bladder cancer found among workers of this industry in several countries.

In the previous IARC evaluation, ${ }^{1}$ the excess risk of leukaemia was considered to be real and was attributed to exposure to solvents, particularly benzene. Results from most recent studies tend to confirm this conclusion. The magnitude of the risk varied between studies, with the highest risks found in studies conducted in North America. A variety of solvent mixtures, with or without benzene, have been used in rubber cements, glues, binding, and release agents. As for bladder cancer, these findings suggest that removal of a single agent (benzene) may not eliminate the risk of leukaemia in the entire industry because of widespread exposure to various carcinogens.

Recent studies tend to confirm the presence of a moderate excess risk of lung cancer, and on the whole results are now more consistent than at the time of the IARC evaluation. Relative risks were $>50 \%$ in many cohorts, indicating that the excesses found are unlikely to be due solely to confounding by smoking ${ }^{115}$ or other lifestyle factors. ${ }^{6}$

An excess risk of stomach cancer was identified in earlier studies of workers in the rubber industry, and has been reported in a recent case-control study from the United Kingdom. ${ }^{75}$ Cohort studies reported after 1982 either do not confirm the presence of an excess risk of stomach cancer in this industry or suggest the presence of only a slight excess risk. A significant excess risk was found in the largest workers cohort. ${ }^{24}$ The risk, however, ranged between 1.1 and 1.3, and is well within the margins of an effect of confounding by socioeconomic status. ${ }^{116}$

Findings for other cancer sites, with the exception of laryngeal cancer, are not consistent between studies, or derive from too few studies. Excess risks found in single studies may be related to specific exposure circumstances occurring in particular rubber plants. One of the most important problems, however, in evaluating findings for other cancer sites, is that reporting may have been incomplete in cohort and case-control studies, with possible preferential reporting of positive findings. The overall findings, therefore, do not provide clear support of excess risks for most of the remaining cancers. The available evidence does not allow an evaluation of the specific agents that may be associated with the increased risk for laryngeal cancer in this industry.

Exposure levels to chemical agents in the rubber industry have decreased in recent years, at least in industrialised countries. ${ }^{13}{ }^{14}$ It is consequently plausible that rates of disease among the workers would have also diminished. Existing epidemiological studies, however, have not shown an elimination of an excess cancer risk. There are some indications that risks are higher 
among workers employed in early years and in less developed countries where presumably exposures are less controlled. The few recent studies of workers employed after the 1960s do not have enough power yet to detect potential moderately increased rates of disease. Even these few studies refer to exposure levels in the 1960 s which were different both in level and in type than exposures in modern rubber plants. Also, it should be stressed that exposure patterns in industrialised countries may not be representative of rubber plants in newly developed or developing countries. The sparse available data indicate that exposure levels are higher in industries of such countries where many sectors of this industry, such as tyre production, tend to be transferred. ${ }^{117}$

\section{Recommendations and conclusions}

Existing studies in the rubber industry have shown the presence of a cancer risk. The potential for diminishing this risk by controlling exposures has also been shown. A common characteristic of existing studies is the absence of detailed exposure assessment and the link between specific exposures and specific risks. New epidemiological studies in this industry should preferably rely on cancer incidence rather than mortality statistics and should provide a detailed evaluation of specific occupational exposures. New studies should also focus on new (or newly identified) risks, such as the possible increase in laryngeal cancer. Biochemical epidemiological techniques should be incorporated both for the evaluation of exposure-for example, measuring aromatic amine haemoglobin or DNA adducts - and of possible interactions between genetics and the environment - for example, examining genetic polymorphisms. ${ }^{118119}$ Their role relative to occupational exposures in workers in this industry has been little evaluated.

In conclusion, findings of epidemiological studies in the rubber industry published after 1982 indicate that there is a moderate increase of the risk of neoplasms of the urinary bladder, lung, larynx, and leukaemia. There was a considerable variation of the risk estimates between studies, but the excess risks were found in many countries and in many different departments indicating that they may be causally related to occupational exposures in this industry. Evidence for other cancer sites, including the stomach, is inconsistent and possibly affected by reporting bias. Examination of new cohorts of workers in the rubber industry, incorporating detailed methods for assessment of exposure and examining various genetic susceptibility markers, may contribute to assessing present risks in this industry and associate these risks with specific exposures.

The study was partly supported by Europe Against Cancer grant $96 / \mathrm{CAN} / 47665$ of the European Commission and by the Generalitat of Catalunya (grant CIRIT/1995 SGR 00434). MK was partly supported by a fellowship from the Ministry of Education and Science, Spain (DGICT, SAB95-0189).

1 International Agency for Research on Cancer. Monographs on the evaluation of the carcinogenic risk of chemicals to on the evaluation of the carcinogenic risk of chemicals
humans. Vol 28. The rubber industry. Lyon: IARC, 1982.
2 International Agency for Research on Cancer. Monographs on the evaluation of carcinogenic risks to humans. Overall
evaluations of carcinogenicity. An updating of IARC monoevaluations of carcinogenicity. An updating of IARC
graphs. Vols 1-42 (suppl 7). Lyon: IARC, 1987.

3 Case RAM, Hosker ME, McDonald DB, Pearson JD Tumors of the urinary bladder in workmen engaged in the manufacture and use of certain dyestuff intermediates in the British chemical industry. Br F Ind Med 1954;11:75104.

4 Davies J. Bladder tumours in the electric-cable industry. Lancet 1965; ii: 143-6.

5 Veys C. Bladder cancer in rubber workers: the story reviewed and updated. Plastics Rubber Process Appl 1981;1: 207-12.

6 Veys CA. Towards causal inference in occupational cancer epidemiology-I. An example of the interpretive value of using local rates as the reference statistic. Ann Occup Hyg 1990;34:349-59.

7 Baxter P, Werner J. Mortality in the British rubber industries 1967-76. London: Her Majesty's Stationery Office, 1980.

8 McMichael A, Spirtas R, Kupper L. An epidemiologic study of mortality within a cohort of rubber workers, 1964-72. $\mathcal{F}$ Occup Med 1974;16:458-64.

9 Andjelkovich D, Taulbee J, Symons M. Mortality experience of a cohort of rubber workers, 1964-73. F Occup Med 1976;18:387-94

10 McMichael A, Spirtas R, Gamble J, Tousey P. Mortality among rubber workers: relationship to specific jobs. $\mathcal{f}$ Occup Med 1976;18:178-85.

11 Goldsmith D, Smith A, McMichael A. A case-control study of prostate cancer within a cohort of rubber and tire workers. F Occup Med 1980;22:533-41.

12 McMichael A, Spirtas R, Kupper L, Gamble J. Solvent exposure and leukemia among rubber workers: an epidemiologic study. I Occup Med 1975;17:234-9.

13 Kromhout H, Swuste P, Boleij JSM. Empirical modelling of chemical exposure in the rubber manufacturing industry. Ann Occup Hyg 1994;38:3-22.

14 Swuste P, Kromhout H. Improving working conditions in the rubber manufacturing industry in The Netherlands. Occupational Hygiene 1996;3:341-9.

15 Holmberg B, Westerholm P, Maasing R, et al. Retrospective cohort study of two plants in the Swedish rubber industry. Scand $\mathcal{F}$ Work Environ Health 1983;9:59-68.

16 Gustavsson P, Hogstedt C, Holmberg B. Mortality and incidence of cancer among Swedish rubber workers, 1952-81. Scand 7 Work Environ Health 1986;12:538-44.

17 Norseth T, Andersen A, Giltvedt J. Cancer incidence in the rubber industry in Norway. Scand f Work Environ Health rubber industry

18 Delzell E, Monson R. Mortality among rubber workers: VIII. Industrial products workers. Am f Ind Med 1984;6: 273-9.

19 Delzell E, Monson R. Mortality among rubber workers: VII. Aerospace workers. Am F Ind Med 1984;6:265-71.

20 Delzell E, Monson R. Mortality among rubber workers: IX. Curing workers. Am f Ind Med 1985;8:537-44.

21 Delzell E, Monson R. Mortality among rubber workers: X. Reclaim workers. Am f Ind Med 1985;7:307-13.

22 Wang $\mathrm{H}$, You $\mathrm{X}, \mathrm{Qu} \mathrm{Y}$, et al. Investigation of cancer epidemiology and study of carcinogenic agents in the Shangai rubber industry. Cancer Res 1984;44:3101-5.

23 Sorahan T, Parkes H, Veys C, Waterhouse J. Cancer mortality in the British rubber industry: 1946-80. Br f Ind Med 1986;43:363-73.

24 Sorahan T, Parkes H, Veys C, Waterhouse J, Straughan J, Nutt A. Mortality in the British rubber industry 1946-85. Br f Ind Med 1989;46:1-11.

25 Veys CA. Bladder cancer as an occupational disease in the British rubber industry: an in-depth factory study to show the past extent of the risk and confirmation of its subsequent disappearance. Progress in Rubber and Plastics Technology 1992;8:1-14.

26 Veys CA. Bladder cancer in rubber workers. Progress in Rubber and Plastics Technology 1995;11:86-7.

27 Bernardinelli L, De Marco R, Tinelli C. Cancer mortality in an Italian rubber factory. $\mathrm{Br}$ F Ind Med 1987;44:187-91

28 Negri E, Piolatto G, Pira E, Decarli A, Kaldor J, La Vecchia C. Cancer mortality in a northern Italian cohort of rubber workers. Br F Ind Med 1989;46:624-8.

29 Zhang Z, Yu S, Li W, Choi B. Smoking, occupational exposure to rubber, and lung cancer. Br F Ind Med 1989;46:125 .

30 Szeszenia-Dabrowska N, Wilczynska U, Kaczmarek T, Szymczak W. Cancer mortality among male workers in the Polish rubber industry. Polish fournal of Occupational Medicine and Environmental Health 1991;4:149-57.

31 Carlo G, Jablinske M, Lee N, Sund K, Corn M. Reduced mortality among workers at a rubber plant. $\mathcal{F}$ Occup Med 1993;35:611-6.

32 Solionova L, Smulevich V. Mortality and cancer incidence in a cohort of rubber workers in Moscow. Scand $\mathcal{F}$ Work Environ Health 1993;19:96-101

33 Weiland S, Mundt K, Keil U, et al. Cancer mortality among workers in the German rubber industry: 1981-91. Осcup Environ Med 1996;53:289-98.

34 Choi B, Nethercott J. A proportionate mortality study on risk of bladder cancer among rubber workers. Cancer Detect Prev 1991;15:403-6.

35 Wilcosky T, Checkoway H, Marshall E, Tyroler H. Cancer mortality and solvent exposures in the rubber industry. $\mathrm{Am}$ Ind Hyg Assoc f 1984;45:809-11. 
36 Arp EW, Wolf PH, Checkoway H. Lymphocytic leukemia and exposures to benzene and other solvents in the rubber industry. F Occup Med 1983;25:598-602.

37 Delzell E, Andjelkovich D, Tyroler H. A case-control study of employment experience and lung cancer among rubber workers. Am f Ind Med 1982;3:393-404.

38 Andjelkovich D, Abdelghany N, Mathew R, Blum S. Lung cancer case-control study in a rubber manufacturing plant. Am F Ind Med 1988;14:559-74.

39 Bourguet CC, Checkoway H. A case-control study of skin cancer in the tire and rubber manufacturing industry. $A m \mathcal{F}$ Ind Med 1987;11:461-73.

40 Symons MJ, Andjelkovich DA, Spirtas R, et al. Brain and central nervous system cancer mortality in US rubber central nervous system cancer mortality in
workers. Ann NY Acad Sci $1982 ; 381: 146-59$.

41 Schoenberg J, Stemhagen A, Mogielnicki A, Altman R, Abe $\mathrm{T}$, Mason T. Case-control study of bladder cancer in New Jersey. I. Occupational exposures in white males. $f$ Nat Cancer Inst 1984;72:973-81.

42 Vineis P, Magnani C. Occupation and bladder cancer in males: a case-control study. Int f Cancer 1985;35:599-606.

43 Morrison AS, Ahlbom A, Verhoek WG, et al. Occupation and bladder cancer in Boston, USA, Manchester, UK, and Nagoya, Japan. F Epidemiol Community Health 1985;39: 294-300.

44 Hoar-Zahm S, Hartge P, Hoover R. The national bladder cancer study: Employment in the chemical industry. $7 \mathrm{Natl}$ Cancer Inst 1987:79:217-22.

45 Jensen OM, Wahrendorf J, Knudsen JB, Sorensen BL. The Copenhagen case-referent study on bladder cancer. Risks among drivers, painters and certain other occupations. Scand $\mathcal{F}$ Work Environ Health 1987;13:129-34.

46 Schifflers E, Jamart J, Renard V. Tobacco and occupation as risk factors in bladder cancer: a case-control study in southern Belgium. Int $\mathcal{F}$ Cancer 1987;39:287-92.

47 Iscovich J, Castelletto R, Esteve J, et al. Tobacco smoking, occupational exposure and bladder cancer in Argentina. Int f Cancer 1987;40:734-40.

48 Risch HA, Burch JD, Miller AB, Hill GB, Steele R, Howe GR. Occupational factors and the incidence of cancer of GR. Occupational factors and the incidence of can

49 Claude JC, Frentzel-Beyme RR, Kunze E. Occupation and risk of cancer of the lower urinary tract among men. A case control study. Int $\mathcal{F}$ Cancer $1988 ; 41: 371-9$.

50 Claude J, Kunze E, Frentzel-Beyme R, et al. Life-style and occupational risk factors in cancer of the lower urinary tract. Am ₹ Epidemiol 1986;124:578-89.

51 Kunze E, Chang-Claude J, Frentzel-Beyme R. Life style and occupational risk factors for bladder cancer in Germany. A case-control study. Cancer 1992;69:1776-90.

52 González CA, López-Abente G, Errezola M, et al. Occcupation and bladder cancer in Spain: a multi-centre casecontrol study. Int f Epidemiol 1989;18:569-77.

53 La Vecchia C, Negri E, D’Avanzo B, Franceschi S. Occupation and the risk of bladder cancer. Int $f$ Epidemiol $1990 ; 19 \cdot 264-8$

54 Notani PN, Shah P, Jayant K, Balakrishnan V. Occupation and cancers of the lung and bladder: a case-control study in Bombay. Int f Epidemiol 1993;22:185-201.

55 Cordier S, Clavel J, Limasset JC, et al. Occupational risks of bladder cancer in France: a multicentric case-control study. Int $\mathcal{F}$ Epidemiol 1993;22:403-11.

56 Hours M, Dananche B, Fevotte J, et al. Bladder cancer and occupational exposures. Scand $\mathcal{F}$ Work Environ Health 1994; 20:322-30.

57 Barbone F, Franceschi S, Talamini R, et al. Occupation and bladder cancer in Pordenone (north-east Italy): a casecontrol study. Int $\mathcal{F}$ Epidemiol 1994;23:58-65.

58 Swanson GM, Burns PB. Cancer incidence among women in the workplace: A study of the association between occupation and industry and 11 cancer sites. $f$ Occup Med 1995;37:282-7.

59 Bernard SM, Cartwright RA, Darwin CM, et al. Hodgkin's disease: case control epidemiological study in Yorkshire. $\mathrm{Br}$ f Cancer 1987;55:85-90

60 Persson B, Dahlander AM, Fredriksson M, et al. Malignant ymphomas and occupational exposures. $\mathrm{Br} \mathcal{F}$ Ind $\mathrm{Med}$ 1989;46:516-20.

61 La Vecchia C, Negri E, D'Avanzo B, Franceschi S. Occupation and lymphoid neoplasms. Brf Cancer 1989;60:385-8.

62 Franceschi S, Serraino D, La Vecchia C, et al. Occupation and risk of Hodgkin's disease in north-east Italy. Int f Cancer 1991;48:831-5.

63 Blair A, Linos A, Stewart PA, et al. Evaluation of risks for non-Hodgkin's lymphoma by occupation and industry exposures from a case-control study. $A m$ f Ind $M e d$ 1993;23:301-12

64 Pottern LM, Heineman EF, Olsen JH, et al. Multiple myeloma among Danish women: employment history and workplace exposures. Cancer Causes Control 1992;3:42732.

65 Demers PA, Vaughan TL, Koepsell TD, et al. A case-control study of multiple myeloma and occupation. Am F Ind Med 1993;23:629-39.

66 Flodin U, Fredriksson M, Persson B, Axelson O. Chronic lymphatic leukemia and engine exhausts, fresh wood, and DDT: a case-referent study. Br f Ind Med 1988;45:33-8.

67 Malone KE, Koepsell TD, Daling JR, et al. Chronic lymphocytic leukemia in relation to chemical exposures. Am f Epidemiol 1989;130:1152-8.

68 Kjuus H, Skjaerven R, Langard S, et al. A case-referent study of lung cancer, occupational exposures and smoking. Scand $\mathcal{f}$ Work Environ Health 1986;12:193-202.
69 Schoenberg JB, Stemhagen A, Mason TJ, et al. Occupation and lung cancer risk among New Jersey white males. F Natl Cancer Inst 1987;79:13-21.

70 Levin LI, Zheng W, Blot WJ, et al. Occupation and lung cancer in Shanghai: a case control study. $\mathrm{Br} \mathcal{F}$ Ind Med 1988;45:450-8.

71 Burns PB, Swanson GM. The occupational cancer incidence surveillance study (OCISS): risk of lung cancer by usual occupation and industry in the Detroit metropolitan area. Am f Ind Med 1991;19:655-71.

72 Jöckel $\mathrm{KH}$, Ahrens W, Wichmann HE, et al. Occupational and environmental hazards associated with lung cancer. Int f Epidemiol 1992;2:202-13.

73 Brownson RC, Alavanja MCR, Chang JC. Occupational risk factors for lung cancer among non-smoking women: a case control study in Missouri (United States). Cancer Causes Control 1993;4:449-54.

74 Wu-Williams AH, Xu ZY, Blot WJ, Dai XD, et al. Occupation and lung cancer risk among women in northern China. Am f Ind Med 1993;24:67-79.

75 Coggon D, Barker DJP, Cole RB. Stomach cancer and work in dusty industries. Br f Ind Med 1990;47:298-301.

76 Gonzalez CA, Sanz M, Marcos G, et al. Occupation and gastric can

77 Cocco P, Palli D, Buiatti E, et al. Occupational exposures as risk factors for gastric cancer in Italy. Cancer Causes Control 1994;5:241-8.

78 Peters RK, Garabrant DH, Yu MC, et al. A case-control study of occupational and dietary factors in colorectal cancer in young men by subsite. Cancer Res 1989;49:5459-68.

79 Zagraniski RT, Kelsey JL, Walter SD. Occupational risk factors for laryngeal carcinoma: Connecticut, 1975-80. Am f Epidemiol 1986;124:67-76.

80 Muscat JE, Wynder EL. Tobacco, alcohol, asbestos, and occupational risk factors for laryngeal cancer. Cancer 1992; 69:2244-51.

81 Elghany NA, Schumacher MC, Slattery ML, et al. Occupation, cadmium exposure, and prostate cancer. Epidemiology 1990;1:107-15.

82 LeMarchand L, Kolonel LN, Yoshizawa CN. Lifetime occupational physical activity and prostate cancer risk. $\mathrm{Am}$ f Epidemiol 1991;133:103-11.

83 McLaughlin JK, Blot WJ, Mandel JS, et al. Etiology of cancer of the renal pelvis. $\mathcal{F}$ Natl Cancer Inst 1983;71:287-91.

84 Partanen T, Heikkila P, Hernberg S, et al. Renal cell cancer and occupational exposure to chemical agents. Scand $\mathcal{F}$ Work Environ Health 1991;17:231-9.

85 Burch JD, Craib KJP, Choi BCK, et al. An exploratory casecontrol study of brain tumors in adults. F Natl Cancer Inst 1987;78:601-9.

86 Preston-Martin S, Mack W, Henderson BE. Risk factors for gliomas and meningiomas in males in Los Angeles county. 989;49:6137-43.

87 Merletti F, Boffetta P, Ferro G, et al. Occupation and cancer of the oral cavity or oropharynx in Turin, Italy. Scand $\mathcal{F}$ Work Environ Health 1991;17:248-54.

88 Roush GC, Walrath J, Stayner LT, et al. Nasopharyngeal cancer, sinonasal cancer, and occupations related to formaldehyde: a case-control study. $\mathcal{F}$ Natl Cancer Inst 1987;79:1221-4.

89 Huebner W, Schoenberg J, Kelsey J, et al. Oral and pharyngeal cancer and occupation: a case-control study. Epidemiology 1992;3:300-9.

90 Serraino D, Franceschi S, La Vecchia C, Carbone A. Occupation and soft-tissue sarcoma in northeastern Italy. Cancer Causes Control 1992;3:25-30.

91 Norell S, Ahlbom A, Lipping H, Österblom L. Oesophageal cancer and vulcanisation work. Lancet 1983;i:462-3.

92 Malker H, McLaughlin J, Silverman D, et al. Occupational risks for bladder cancer among men in Sweden. Cancer Res 1987;47:6763-6.

93 Olsen JH, Jensen OM. Occupation and risk of cancer in Denmark. An analysis of 93810 cancer cases, 1970-9. Scand F Work Environ Health 1987;13(suppl 1):1-91.

94 Zheng W, McLaughlin JK, Gao YT, Silverman DT, Gao $\mathrm{RN}$, Blot WJ. Bladder cancer and occupation in Shanghai, 1980-4. Am f Ind Med 1992; 21:877-85.

95 Dolin PJ, Cook-Mozaffari P. Occupation and bladder (1992;66:568 78.

96 Linet MS, Malker HRS, McLaughlin JK, et al. NonHodgkin's lymphoma and occupation in Sweden: a registry based analysis. Br F Ind Med 1993;50:79-84.

97 Pukkala E. Cancer risk by social class and occupation. A survey of 109000 cancer cases among Finns of working age. Basel: Karger, 1995.

98 Hrubec Z, Blair AE, Vaught J. Mortality risks by industry among US veterans of known smoking status 1954-1980. Bethesda, MD: US DHHS, 1995. (NIH Publ No 95-2747.)

99 Costa G, Faggiano F, Lagorio S. Occupational mortality in Italy in the 80s. Rome: Istituto Superiore per la Prevenzione e la Sicurezza del Lavoro, 1995.

100 Coggon D, Pannett B, Acheson DE. Use of job-exposure matrix in an occupational analysis of lung and bladder cancers on the basis of death certificates. $\mathcal{F}$ Natl Cancer Inst $1984 ; 72: 61-5$.

101 Baxter PJ, McDowall ME. Occupation and cancer in London: an investigation into nasal and bladder cancer using the cancer atlas. Br f Ind Med 1986;43:44-9.

102 Gallagher RP, Threlfall WJ, Band PR, Spinelli JJ. Occupational mortality in British Columbia 1950-84. Vancouver: 
Cancer Control Agency of British Columbia and the Workers' Compensation Board of BC, 1989.

103 Hall N, Rosenman K. Cancer by industry: analysis of a population-based cancer registry with an emphasis on blue-collar workers. Am f Ind Med 1991;19:145-59.

104 Burnett CA, Silverman DT, Lalich NR. A comparison of analyses of occupational bladder cancer death certificates $v$ population-based case-control interview data. $A m \mathcal{F}$ Ind Med 1994;25:677-88.

105 Roman E, Carpenter L. Cancer incidence in England 1981-7. In: Drever F, ed. Occupational Health. Decennial Supplement. London: HMSO, 1995.

106 Schumacher MC, Delzell E. A death-certificate casecontrol study of non-Hodgkin's lymphoma and occupation in men in North Carolina. Am F Ind Med 1988;13:317-30.

107 Heineman EF, Olsen JH, Pottern LM, et al. Occupational risk factors for multiple myeloma among Danish men.
Cancer Causes Control 1992;3:555-68.

108 Figgs LW, Dosemeci M, Blair A. Risk of multiple myeloma by occupation and industry among men and women: 24-state death certificate study. F Occup Med 1994;36: 24-state

109 Dubrow R, Wegman DH. Occupational characteristics of cancer victims in Massachusetts 1971-3. Cincinatti, US DHSS, NIOSH, 1984. (Publ No 84-109.)

110 Loomis DP, Savitz DA. Occupation and leukemia mortality among men in 16 states: 1985-7. Am f Ind Med 1991;19:509-21.

111 Milne KL, Sandler DP, Everson RB. Lung cancer and occupation in Alameda county: a death certificate casecontrol study. Am f Ind Med 1983;4:565-75.
112 Wang Q, Boffetta P, Parkin DM, Kogevinas M. Occupational risk factors for lung cancer in Tianjin, China. Am 7 Ind Med 1995;28:353-62.

113 Ward MH, Dosemeci M, Cocco P. Mortality from gastric cardia and lower esophagus cancer and occupation. F Occup Med 1994;36:1222-7.

114 Wong O, Raabe GK. Application of meta-analysis in reviewing occupational cohort studies. Occup Environ Med 1996;53:793-800.

115 Siemiatycki J, Gérin M, Steward P, Nadon L, Dewar R, Richardson L. Associations between several sites of cancer and 10 types of exhaust and combustion products. Scand $\mathcal{F}$ Work Environ Health 1988;14:79-90.

116 Faggiano F, Partanen T, Kogevinas M, Boffetta P. Socioeconomic differences in cancer incidence and mortality. In: Kogevinas M, Pearce N, Susser M, Boffetta P, eds. Social inequalities in cancer. Lyon: International Agency for Research on Cancer, 1997. (IARC Sci Publ No 138.)

117 Kogevinas M, Boffetta P, Pearce N. Occupational exposure to occupational carcinogens in developing countries. In: Pearce N, Matos E, Vainio H, Boffetta P, Kogevinas M, eds. Occupational cancer in developing coutries. Lyon: InterOccupation cancer in developing countries. Lyon: Inter(IARC Sci Publ No 129.)

118 Vineis P, Terracini B. Biochemical epidemiology of bladder cancer. Epidemiology 1990;1:448-52.

119 Brockmoller J, Kerb R, Drakoulis N, Staffeldt B, Roots I. Glutathione S-transferase $M 1$ and its variants $A$ and $B$ as host factors of bladder cancer susceptibility: a case-control study. Cancer Res 1994;54:4103-11.

\section{Medical editors' trial amnesty}

As described in an editorial in the British Medical fournal, ${ }^{1}$ medical editors of nearly 100 international medical journals are taking action to try to ensure that the results of unpublished randomised controlled trials become available to be included in systematic reviews. This could have important benefits for patient care.

Any reader who would like to take up this opportunity to register the results of a trial that did not getpublished can do so on a special unreported trial registration form. Copies are available from the Occupationaland Environmental Medicine editorial office.

I do not expect that many Occupational and Environmental Medicine readers will need to take up this offer, given the nature of our field, but perhaps I will be proved wrong.

ANNE COCKCROFT

Editor

1 R Smith, I Roberts. An amnesty for unpublished trials. BMF 1997;315:622. 